\title{
EFFECT OF CHANNEL ESTIMATION ERROR ON THE PERFORMANCE OF SVD-BASED MIMO COMMUNICATION SYSTEMS
}

\author{
Alejandro Cano Gutiérrez ${ }^{1,2}$, Milica Stojanovic ${ }^{2}$ \\ ${ }^{1}$ Universitat Politècnica de Catalunya, Jordi Girona 1-308034 Barcelona, Spain, afcano@mit.edu \\ ${ }^{2}$ Massachusetts Institute of Technology, 77 Massachusetts Av, Cambridge, MA 02139-4307, USA, militsa@mit.edu
}

\begin{abstract}
Recent information-theoretic work has shown the potential capacity increase of Multiple-input Multipleoutput (MIMO) systems compared to Single-input Singleoutput (SISO) systems [1] [2]. The Singular Value Decomposition (SVD) has proven very useful in MIMO communication systems but its performance depends on the accuracy of the Channel State Information (CSI) at both sides of the link. This paper presents a study on the influence of channel estimation errors and noise on the probability of error of the system when no error correction mechanisms are provided.
\end{abstract}

Keywords - MIMO, SVD, Singular Value Decomposition, Wireless Communication Systems.

\section{INTRODUCTION AND NOTATION}

A linear Multiple-input Multiple-output (MIMO) system with $N_{t}$ inputs (transmitters), $\mathbf{x}=\left(x_{1} \ldots x_{N_{t}}\right)^{T}$, and $N_{r}$ outputs (receivers), $\mathbf{y}=\left(y_{1} \ldots y_{N_{r}}\right)^{T}$, can be characterized by the following expression

$$
\left(\begin{array}{c}
y_{1} \\
\vdots \\
y_{N_{r}}
\end{array}\right)=\mathbf{A}\left(\begin{array}{c}
x_{1} \\
\vdots \\
x_{N_{t}}
\end{array}\right)+\left(\begin{array}{c}
n_{1} \\
\vdots \\
n_{N_{r}}
\end{array}\right)
$$

where $\mathbf{n}=\left(n_{1} \ldots n_{N_{r}}\right)^{T}$ are noise samples and the channel matrix A can be either constant, in the case of a frequency non-selective channel, or a function in the frequency domain, in the case of a frequency-selective channel.

In the following analysis, we will consider that the number of receive antennas is at least the number of transmit antennas, so that, in the case where the channel matrix is full rank, all the eigenmodes can be decoded.

The Singular Value Decomposition (SVD) of a rank$r$ matrix $\mathbf{A}$ is $\mathbf{A}=\mathbf{U} \mathbf{\Sigma} \mathbf{V}^{\mathbf{H}}$, where $\mathbf{U}$ and $\mathbf{V}$ are unitary matrices, $(\cdot)^{H}$ denotes conjugate transpose and $\boldsymbol{\Sigma}=\operatorname{diag}\left(\sigma_{1} \ldots \sigma_{r}\right)$, where $\sigma_{1} \geq \ldots \geq \sigma_{r}>0$ are the channel singular values [3]. In the case of a frequency selective channel, they would be defined for every frequency. The importance of SVD in MIMO communication systems relies on the fact that the presteering matrix $\mathbf{V}$ at the transmitter and the steering matrix $\mathbf{U}^{H}$ at the receiver decompose the MIMO channel into $r$ SISO orthogonal modes of excitation or eigenchannels [4].

$$
\mathbf{U}^{\mathbf{H}} \mathbf{y}=\mathbf{U}^{\mathbf{H}} \mathbf{A V} \mathbf{x}+\mathbf{U}^{\mathbf{H}} \mathbf{n}=\mathbf{\Sigma} \mathbf{x}+\mathbf{U}^{\mathbf{H}} \mathbf{n}
$$

Let us suppose that the estimate of the channel matrix is slightly different from the actual channel matrix by an amount defined as an additive perturbation matrix $\mathbf{E}$; that is, the estimated channel matrix is $\tilde{\mathbf{A}}=\mathbf{A}+\mathbf{E}$. This estimation error can be the result of both quantization error and time variation of the channel. We want the influence of this error matrix to be low enough not to change the decisions of the decoder. To this end, we will consider an 8-PSK constellation and we will ignore the influence of coding. In this situation,

$$
\widehat{\mathbf{X}}=\boldsymbol{\Sigma}^{-1} \mathbf{U}^{\mathbf{H}}(\tilde{\mathbf{A}} \tilde{\mathbf{X}}+\mathbf{N}), \quad \tilde{\mathbf{X}}=\mathbf{V X}
$$

Therefore, the error function for each component, $\Upsilon=$ $\widehat{x_{i}}-x_{i}$, can be expressed as

$$
\Upsilon=\frac{1}{\sigma_{i}} \mathbf{u}_{\mathbf{i}}^{\mathbf{H}}\left(\begin{array}{ccc}
\uparrow & & \uparrow \\
\mathbf{e}_{\mathbf{1}} & \ldots & \mathbf{e}_{\mathbf{N}_{\mathbf{t}}} \\
\downarrow & & \downarrow
\end{array}\right)\left(\begin{array}{ccc}
\uparrow & & \uparrow \\
\mathbf{v}_{\mathbf{1}} & \ldots & \mathbf{v}_{\mathbf{N}_{\mathbf{t}}} \\
\downarrow & & \downarrow
\end{array}\right) \mathbf{x}+\frac{\widehat{n}_{i i}}{\sigma_{i}}
$$

where $\widehat{\mathbf{n}}=\mathbf{U}^{\mathbf{H}} \mathbf{n}$ has the same statistic properties as $\mathbf{n}$ for $\mathbf{U}$ being a unitary matrix. Let us define $\epsilon_{\mathbf{i}}=\mathbf{u}_{\mathbf{i}}^{\mathbf{H}} \mathbf{E}$. Then

$$
\Upsilon=\frac{1}{\sigma_{i}}\left(\epsilon_{\mathbf{i}} \mathbf{V} \mathbf{x}+\widehat{n}_{i i}\right)
$$

We will not have a decoding error if the error component $\Upsilon$ makes $\widehat{x}_{i}$ remain in the region assigned to $x_{i}$ by the decoder (see Figure 1). Without loss of generality we can assume that $x_{i}=1$. Then, we can express this condition mathematically in the following way

$$
\begin{gathered}
\left\{\begin{array}{l}
\left\langle\Upsilon, S_{1}\right\rangle<\sin \left(\frac{\pi}{8}\right) \\
\left\langle\Upsilon, S_{2}\right\rangle<\sin \left(\frac{\pi}{8}\right)
\end{array}\right. \\
S_{1}=\left(\cos \left(\frac{5 \pi}{8}\right), \sin \left(\frac{5 \pi}{8}\right)\right)=\left(-\sin \left(\frac{\pi}{8}\right), \cos \left(\frac{\pi}{8}\right)\right) \\
S_{2}=\left(\cos \left(\frac{-5 \pi}{8}\right), \sin \left(\frac{-5 \pi}{8}\right)\right)=\left(-\sin \left(\frac{\pi}{8}\right),-\cos \left(\frac{\pi}{8}\right)\right)
\end{gathered}
$$

where the function $\langle\cdot, \cdot\rangle$ is the usual $\mathbb{R}^{2}$-scalar product, $<a, b>=\sum a_{i} b_{i}$. If $a$ or $b$ are complex variables, they will be substituted by their image in the real space, via the canonical isometry. 


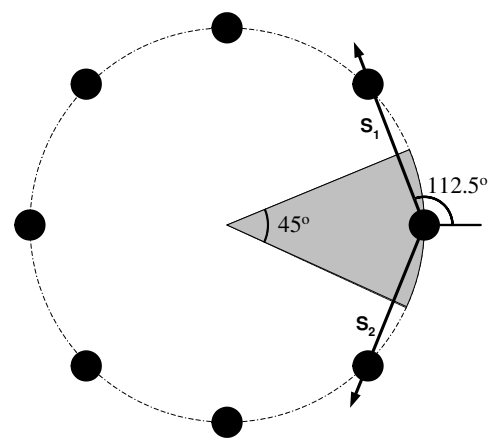

Fig. 1

It is not possible to decouple the influence of channel variation and that of the noise nor treat them jointly unless we do some assumptions or assign values to the parameters in the system. Therefore, a study of the two extreme cases, i.e., the case when the influence of the noise term is dominant and the case when its influence is negligible, will be carried out. Later on, a joint study will be done under certain assumptions.

\section{THE NOISE TERM IS DOMINANT}

In this case

$$
\Upsilon=\widehat{x}_{i}-x_{i}=\frac{\widehat{n}_{i i}}{\sigma_{i}}
$$

and we must guarantee Equation (5). If we define the signal to noise ratio of the $i t h$ channel as $S N R_{i}=\mathbf{E}\left(\frac{\sigma_{i}^{2}}{\widehat{n}_{i i}^{2}}\right)=\frac{\sigma_{i}^{2}}{\sigma_{n}^{2}}$, the performance of the channel will be good if

$$
\frac{1}{\sqrt{S N R_{i}}} \max \left(\left\langle\alpha, S_{1}\right\rangle,\left\langle\alpha, S_{2}\right\rangle\right)<\sin \left(\frac{\pi}{8}\right)
$$

where $\alpha=\alpha_{R}+i \alpha_{I}$ is a sample from a circularly symmetric complex Gaussian random variable with variance 1. The above inequality can be rewritten as

$$
\Theta=-\alpha_{R}+\max \left( \pm \alpha_{I} \cot \left(\frac{\pi}{8}\right)\right)<\sqrt{S N R_{i}}
$$

By means of simulation, the distribution of $\Theta$ has been found. The probability of symbol error is the probability that $\Theta<\frac{\sigma_{i}}{\sigma_{\sigma}}$. Figure 2 shows the probability of error as a function of $\frac{\sigma_{i}}{\sigma_{n}}$ in semilogarithmic axis.

In Table 1, the necessary signal to noise ratios for probabilities of error comprised in $\left[10^{-6}, 10^{-1}\right]$ are given. Besides, a third order interpolating polynomial has been used to extrapolate the values that would be needed for higher reliability.

\section{THE ESTIMATION ERROR IS DOMINANT}

In this case,

$$
\Upsilon=\frac{1}{\sigma_{i}}\left(\epsilon_{\mathbf{i}} \mathbf{V} \mathbf{x}\right)
$$

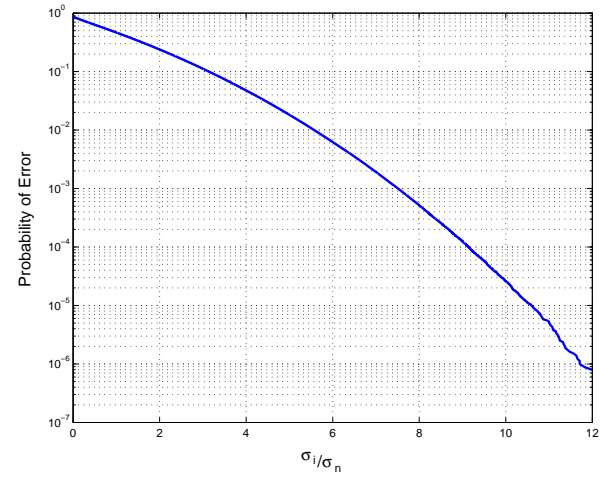

Fig. 2

Table 1

Required values of $S N R_{i}$ for some error probabilities.

\begin{tabular}{|c||c|}
\hline$\tilde{P}$ & $S N R_{i}(d B)$ \\
\hline \hline $10^{-1}$ & 9.9341 \\
$10^{-2}$ & 14.928 \\
$10^{-3}$ & 17.504 \\
$10^{-4}$ & 19.188 \\
$10^{-5}$ & 20.509 \\
$10^{-6}$ & 21.428 \\
$10^{-7}$ & 22.051 \\
$10^{-8}$ & 22.692 \\
$10^{-9}$ & 23.242 \\
$10^{-10}$ & 23.723 \\
\hline
\end{tabular}

Since $\mathbf{V}^{\mathbf{H}}$ is a unitary matrix, its columns form a basis of $\mathbb{C}^{N_{t}}$ so we can write $\epsilon_{i}=\delta_{1} v_{1}^{H}+\delta_{2} v_{2}^{H}+\ldots+\delta_{N_{t}} v_{N_{t}}^{H}$.

$$
\Upsilon=\frac{1}{\sigma_{i}} \delta \mathbf{x}=\frac{1}{\sigma_{i}}\left(\delta_{1} x_{1}+\ldots+\delta_{N_{t}} x_{N_{t}}\right)
$$

\section{A. General approach}

Let us study necessary conditions for the first inequality in Equation (5) to hold. Since $x_{j}, j \neq i$ are independent from $x_{i}$ and the elements $\delta_{k}$ are also out of our control, one possible approach is to find an upper bound over all the possible combinations of those parameters for a given length $\left\|\epsilon_{\mathbf{i}}\right\|=\|\delta\|=K$ of the vector $\epsilon_{\mathbf{i}}$.

$$
\begin{aligned}
& \max _{\left\|\epsilon_{\mathbf{i}}\right\|=K}\left\langle\Upsilon, S_{1}\right\rangle=\max _{\left\|\epsilon_{\mathbf{i}}\right\|=K}\left\langle\frac{1}{\sigma_{i}} \delta \mathbf{x}, S_{1}\right\rangle= \\
& \max _{\tilde{\delta} \in \mathcal{S}^{N_{t}-1}(\mathbb{C})} \frac{K}{\sigma_{i}}\left\langle\tilde{\delta} \mathbf{x},\left(-\sin \left(\frac{\pi}{8}\right), \cos \left(\frac{\pi}{8}\right)\right)\right\rangle
\end{aligned}
$$

By using Lagrange multipliers it is easy to show that the result of this maximization problem is $\frac{K}{\sigma_{i}} \sqrt{N_{t}}$. So, a sufficient condition for the first inequality in (5) to hold is

$$
\frac{\sqrt{N_{t}}\left\|\epsilon_{i}\right\|}{\sigma_{i}}<\sin \frac{\pi}{8} \Leftrightarrow\left\|\epsilon_{i}\right\|<\frac{\sin \frac{\pi}{8}}{\sqrt{N_{t}}} \sigma_{i}=\nu_{0} \sigma_{i}
$$

If we perform the same analysis for the second inequality in (5), the above condition on the perturbation matrix is again obtained. 
Let us recall the definition of $\epsilon_{\mathbf{i}}$

$$
\epsilon_{\mathbf{i}}=\mathbf{u}_{\mathbf{i}}{ }^{H} \mathbf{E}
$$

Since $\mathbf{U}$ is unitary, we can write $\mathbf{e}_{\mathbf{i}}=e_{i}^{1} \mathbf{u}_{\mathbf{1}}+\ldots+e_{i}^{N_{r}} \mathbf{u}_{\mathbf{N}_{\mathbf{r}}}$ and $\epsilon_{i}=\left(e_{1}^{i}, e_{2}^{i}, \ldots, e_{N_{t}}^{i}\right)$. Therefore, if we take the square of (12), we obtain

$$
\sum_{j=1}^{N_{t}}\left(e_{j}^{i}\right)^{2}<\nu_{0}^{2} \sigma_{i}^{2}
$$

In the above expression, we can upper bound the left hand term as

$$
\sum_{j=1}^{N_{t}}\left(e_{j}^{i}\right)^{2} \leq \sum_{j=1}^{N_{t}} \sum_{i=1}^{N_{r}}\left(e_{j}^{i}\right)^{2}=\|\mathbf{E}\|_{F}^{2}
$$

where $\|\cdot\|_{F}$ is the Fröbenius norm of the error matrix. Taking into account that, if the channel matrix is full-rank, the smallest nonzero singular value is $\sigma_{N_{t}}$ and using this expression in (13) we conclude that a sufficient condition for the error matrix is

$$
\|\mathbf{E}\|_{F}<\nu_{0} \sigma_{N_{t}}
$$

Note that the process we have followed so far is only slightly dependent on the constellation. In fact, for any other constellation, we could do the same analysis, the only difference being the value of the constant $\nu_{0}$. A straightforward result of the previous procedure is that $\nu_{0}$ for an N-PSK constellation is $\frac{\sin \left(\frac{\pi}{N}\right)}{\sqrt{N_{t}}}$.

\section{B. Probabilistic approach}

Certainly, extremely pessimistic and possibly unnecessary bounds have been used to provide us with condition (15), which is valid over all the possible realizations of the error matrix. Another approach which provides less stringent conditions is the probabilistic one. In other words, we will find the necessary conditions so that optimal performance-i.e., condition (5) - is not achieved only with a small probability $P_{e}$, which can be written as

$$
\mathcal{P}\left[\left\langle\Upsilon, S_{1}\right\rangle \leq \sin \frac{\pi}{8},\left\langle\Upsilon, S_{2}\right\rangle \leq \sin \frac{\pi}{8} \mid \mathbf{E}\right]>1-P_{e}
$$

A Matlab routine has been developed in order to compute the empiric distribution of the function $\Theta(\mathbf{x}, \delta)=$ $\max \left(\left\langle\delta \mathbf{x},\left(-1, \cot \frac{\pi}{8}\right)\right\rangle,\left\langle\delta \mathbf{x},\left(-1,-\cot \frac{\pi}{8}\right)\right\rangle\right)$ for the case $N_{t}=4, N_{r}=20$. Using this distribution we can express the restriction for $\left\|\epsilon_{i}\right\|$ as

$$
\left\|\epsilon_{i}\right\| \leq \frac{\sigma_{i}}{K_{P_{e}}}
$$

where $K_{P_{e}}$ is the value of $\Theta$ such that only $P_{e}$ of the realizations of $E$ can produce higher values of $\Theta$. Some of the values of $K_{P_{e}}$ are listed in Table 2 .

As it may be expected, $K_{P_{e}}^{-1} \rightarrow \nu_{0}=0.19134$. Another important conclusion of this analysis is that if we have
Table 2

Values of $K_{P_{e}}^{-1}$ and $G_{P_{e}}^{-1}$ for some error probabilities.

\begin{tabular}{|r||c|c|}
\hline$P_{e}(\%)$ & $K_{P_{e}}^{-1}$ & $G_{P_{e}}^{-1}$ \\
\hline \hline 5 & 0.28715 & 1.23717 \\
2.5 & 0.26143 & 1.08520 \\
1 & 0.23982 & 0.94784 \\
0.5 & 0.22885 & 0.87250 \\
0.1 & 0.21293 & 0.74961 \\
0.05 & 0.20856 & 0.71108 \\
0.01 & 0.20171 & 0.63955 \\
0.001 & 0.19651 & 0.56913 \\
0.0001 & 0.19376 & 0.51951 \\
0.00001 & 0.19252 & 0.47995 \\
0.000001 & 0.19185 & 0.46249 \\
\hline
\end{tabular}

means to overcome a certain $P_{e}$, the restrictions over $\mathbf{E}$ can be substantially loosened.

At this point we could proceed as in equation (15) and write

$$
\|\mathbf{E}\|_{F} \leq \frac{\sigma_{i}}{K_{P_{e}}}
$$

Another option, however, is to extend the previous steps to include the random behavior of $\left\|\epsilon_{\mathrm{i}}\right\|$ in the empirical distribution and express the result as a function of $\|\mathbf{E}\|_{F}$. Recall that our goal is that with high probability $\left\|\epsilon_{\mathbf{i}}\right\| \Theta=$ $\|\mathbf{E}\|_{F}\left\|\tilde{\epsilon}_{\mathbf{i}}\right\| \Theta \leq \sigma_{i}$, where $\tilde{\epsilon}_{\mathbf{i}}$ is restricted to the matrices with Fröbenius norm equal to unity. As before, we can use the empirical distribution of $\left\|\tilde{\epsilon}_{\mathbf{i}}\right\| \Theta$ to express a restriction depending on the mean probability of error. This dependency is represented in the function $G_{P e}$, whose definition is analogous to the one given for $K_{P e}$. Therefore, a new condition can be expressed as

$$
\|\mathbf{E}\|_{F} \leq G_{P_{e}}^{-1} \sigma_{i}=\nu_{P_{e}} \sigma_{i}
$$

Some values of $\nu_{P e}$ can be found in Table 2. Note that we obtain an even further loosening in the conditions to be satisfied. Nevertheless, the result for $P_{e}=0$-recall that there is no noise-would still be $\|\mathbf{E}\|_{F} \leq \nu_{0} \sigma_{i}$ since there exist realizations of the channel for which all the inequalities involved in this analysis become equalities.

It should be noted that the conditions obtained herein can be considered from two different standpoints. Indeed, if we know the type of estimation errors for the channel matrix, we can decide how many eigenmodes are suitable for transmitting information along the channel-those for which $\|\mathbf{E}\|_{F} \leq \nu_{P_{e}} \sigma_{i}$. On the other hand, if we are restricted to maintain a fixed number of eigenmodes active, we must monitor $\mathbf{E}$ so that the condition in (19) is valid for all of them and adjust accordingly the design parameters involved such as, for instance, the elapsed time between two consecutive channel estimations.

To continue this analysis we need to suppose a model for the error matrix. Let us assume that we can express the 
temporal channel matrix behavior as $\mathbf{A}(n)=\mathbf{B}(n)+\mathbf{M}$, where the entries of $\mathbf{B}(n)$ are zero mean circularly symmetric complex Gaussian variables with variance $\sigma_{b}^{2}$, and that $\mathbf{B}(n+1)=\alpha \mathbf{B}(n)+\mathbf{W}(n+1)$, where $\alpha$ is a constant modelling the resemblance of the channel matrices in two consecutive symbol times and $\mathbf{W}(n)$ is a matrix of zero mean circularly symmetric complex Gaussian random variables with variance $\sigma_{w}^{2}$. The matrix $\mathbf{M}$ is introduced to account for the fact that, although we assume that the channel matrix elements have zero mean, in a short-term analysis they would have nonzero mean and would be conditioned by the previous temporal evolution.

Under these assumptions, let us define the error matrix $\mathbf{E}(n, s)=\mathbf{A}(n+s)-\mathbf{A}(n)$. It can be easily proven by induction that

$$
\mathbf{E}(n, s)=\left(\alpha^{s}-1\right) \mathbf{B}(n)+\sum_{i=0}^{s-1} \alpha^{i} \mathbf{W}(n+s-i)
$$

Given the fact that the random variables in the sum in the definition of $\mathbf{E}(n, s)$ are zero mean circularly symmetric complex normal random variables, the elements in $\mathbf{E}(n, s)$ are random variables of the same type with variance

$\sigma_{E}^{2}=\left(\alpha^{s}-1\right)^{2} \sigma_{b}^{2}+\sum_{i=0}^{s-1} \alpha^{2 i} \sigma_{w}^{2}=\left(1-\alpha^{s}\right)^{2} \sigma_{b}^{2}+\frac{1-\alpha^{2 s}}{1-\alpha^{2}} \sigma_{w}^{2}$

Furthermore, since $\mathbf{M}$ is constant, and $\mathbf{A}(n)=\mathbf{B}(n)+\mathbf{M}$, $\sigma_{a}=\sigma_{b}$ and

$$
\begin{gathered}
\sigma_{a}^{2}=\mathbf{E}\left\{\left|b_{i j}(n)\right|^{2}\right\}=\mathbf{E}\left\{\left|b_{i j}(n+1)\right|^{2}\right\}= \\
\mathbf{E}\left\{\left(\alpha b_{i j}(n)+w_{i j}(n+1)\right)\left(\alpha b_{i j}(n)+w_{i j}(n+1)\right)^{*}\right\} \\
\sigma_{a}^{2}=\alpha^{2} \sigma_{a}^{2}+\sigma_{w}^{2} \Leftrightarrow\left(1-\alpha^{2}\right) \sigma_{a}^{2}=\sigma_{w}^{2} \\
\sigma_{E}^{2}=\left(1-\alpha^{s}\right)^{2} \sigma_{a}^{2}+\left(1-\alpha^{2 s}\right) \sigma_{a}^{2}=2\left(1-\alpha^{s}\right) \sigma_{a}^{2}
\end{gathered}
$$

Note also that if $X$ is a circularly symmetric normal complex random variable, then $|X|^{2}$ is the sum of the squares of two normal random variables with the same mean and variance. Therefore

$$
\|\mathbf{E}(n, s)\|_{F}^{2}=\sum_{j=1}^{N_{t}} \sum_{i=1}^{N_{r}}\left|e_{i}^{j}\right|^{2}=\sigma_{E}^{2} \sum_{k=1}^{2 N_{t} N_{r}}\left(X_{k}\right)^{2}=\sigma_{E}^{2} \Phi^{2}
$$

where $X_{k}$ are independent identically distributed normal random variables with zero mean and unity variance. Hence, $\Phi^{2}$ follows a chi-squared distribution with $2 N_{t} N_{r}$ degrees of freedom, $\Phi^{2} \sim \chi_{2 N_{t} N_{r}}^{2}$.

Recall that to ensure the proper performance of the decoder we need condition (15) to be satisfied. If we introduce the model for the error matrix, that amounts to

$$
\|\mathbf{E}(n, s)\|_{F}\left\|\tilde{\epsilon_{\mathbf{i}}}\right\| \Theta \leq \sigma_{i} \Rightarrow \Phi\left\|\tilde{\epsilon_{\mathbf{i}}}\right\| \Theta \leq \frac{\sigma_{i}}{\sigma_{E}}
$$

Note that this distribution is directly related to the probability of error of the system since if we guarantee $F_{\Phi \| \tilde{\epsilon_{i} \| \Theta}}^{-1}(1-p) \leq \frac{\sigma_{i}}{\sigma_{E}}$, then the probability of error is less than $p$. Taking into account the fact that $F_{\Phi \| \tilde{\epsilon_{i} \| \Theta}}^{-1}(1-p)$ is a strictly decreasing function of $p$ we can conclude that the probability of error $p$ of the ith channel can be upperbounded as

$$
(1-p) \geq F_{\Phi\left\|\tilde{\epsilon_{i}}\right\| \Theta}\left(\frac{\sigma_{i}}{\sigma_{E}}\right)
$$

$$
p \leq 1-F_{\Phi\left\|\tilde{\epsilon_{i}}\right\| \Theta}\left(\frac{\sigma_{i}}{\sigma_{E}}\right)=1-F_{\Phi\left\|\tilde{\epsilon_{i}}\right\| \Theta}\left(\frac{\sigma_{i}}{\sqrt{2\left(1-\alpha^{s}\right) \sigma_{a}^{2}}}\right)
$$

where $\sigma_{i}$ is the singular value associated to the $i t h$ eigenmode, $\sigma_{a}$ is the variance of the elements in the matrix $\mathbf{A}(n)$ and $\mathrm{s}$ is the number of symbol intervals elapsed since the last estimation of the channel matrix. The next figure shows the dependence of the bound for the probability of error as a function of $\frac{\sigma_{i}}{\sigma_{E}}$ for the case of four transmit antennas and twenty receive antennas.

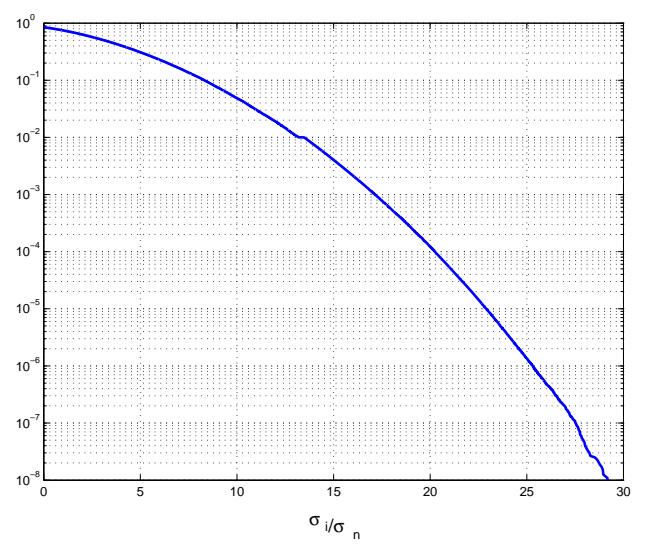

Fig. 3

\section{JOINT STUDY}

Let us expand completely the expression in Equation (4)

$$
\widehat{x}_{i}-x_{i}=\frac{1}{\sigma_{i}}\left[\sum_{j=1}^{N_{t}} \sum_{k=1}^{N_{t}} \sum_{l=1}^{N_{r}} u_{i}^{l} e_{k}^{l} v_{j}^{k} x_{j}+\widehat{n}_{i i}\right]
$$

If we assume as in the previous section that the error matrix can be modelled as $\mathbf{E}(n, s)=$ $\left(\alpha^{s}-1\right) \mathbf{B}(n)+\sum_{i=0}^{s-1} \alpha^{i} \mathbf{W}(n+s-i)$, being $\mathbf{B}$ and $\mathbf{W}$ Gaussian matrices, then all the entries $e_{k}^{l}$ are also samples from a circularly symmetric complex Gaussian distribution with variance $\sigma_{E}$. Hence, the expression above consists of the sum of $N_{t} N_{r}$ zero mean circularly symmetric complex Gaussian random variables whose variance has not been yet determined plus one random variable of the same kind with variance $\sigma_{n}$.

Since the term accompanying each of the $e_{k}^{l}$ is $\sum_{j=1}^{N_{t}} x_{j} u_{i}^{l} v_{j}^{k}$ and all these random variables are independent 
and zero mean normal-distributed, the overall variance of the $N_{t} N_{r}$ variables is

$$
\tilde{\sigma}^{2}=\sigma_{E}^{2} \sum_{k, l}\left|u_{i}^{l} \sum_{j} x_{j} v_{j}^{k}\right|^{2}
$$

If we take the expectation of the expression above with respect to the constellation, keeping in mind that the symbols are assumed independent in this analysis, then

$$
\begin{gathered}
<\tilde{\sigma}^{2}>=\sigma_{E}^{2} \sum_{k, l}\left|u_{i}^{l}\right|^{2} \sum_{j} \sum_{j^{\prime}} \mathbf{E}\left\{x_{j} x_{j^{\prime}}^{*}\right\} v_{j}^{k}\left(v_{j^{\prime}}^{k}\right)^{*}= \\
\sigma_{E}^{2} \sum_{l}\left|u_{i}^{l}\right|^{2} \sum_{k, j}\left|v_{j}^{k}\right|^{2}=\sigma_{E}^{2}\left\|\mathbf{u}_{\mathbf{i}}\right\|^{2}\|\mathbf{V}\|_{F}^{2}=N_{t} \sigma_{E}^{2}
\end{gathered}
$$

Finally, if we include the effect of the noise in this analysis, we obtain

$$
\widehat{x}_{i}-x_{i}=\frac{\sigma}{\sigma_{i}} \xi
$$

where $\xi=\xi_{R}+i \xi_{I}$ is a zero mean circularly symmetric complex random variable with unity variance and

$$
\sigma=\sqrt{N_{t} \sigma_{E}(\alpha, s)^{2}+\sigma_{n}^{2}}=\sqrt{2 N_{t}\left(1-\alpha^{s}\right) \sigma_{a}^{2}+\sigma_{n}^{2}}
$$

Recall that this is the same formulation as that of Section II. In that section, the conditions for correct performance were found to be

$$
\max \frac{\sigma}{\sigma_{i}}\left(\left\langle\xi, S_{1}\right\rangle,\left\langle\xi, S_{2}\right\rangle\right)<\sin \left(\frac{\pi}{8}\right)
$$

If we define $\widehat{S N R_{i}}=\frac{\sigma_{i}^{2}}{\sigma^{2}}$, then the above inequality can be rewritten as

$$
\Theta=-\xi_{R}+\max \left( \pm \xi_{I} \cot \left(\frac{\pi}{8}\right)\right)<\sqrt{\widehat{S N R_{i}}}
$$

Let us define the signal-to-noise ratio at the receiver as

$$
S N R=\frac{\mathbf{E}\left\{(\mathbf{y}-\mathbf{n})^{H}(\mathbf{y}-\mathbf{n})\right\}}{\mathbf{E}\left\{\mathbf{n}^{H} \mathbf{n}\right\}}=\frac{\mathbf{E}\left\{(A V \mathbf{x})^{H}(A V \mathbf{x})\right\}}{\mathbf{E}\left\{\mathbf{n}^{H} \mathbf{n}\right\}}
$$

Taking into account

$$
\begin{aligned}
\mathbf{E}\left\{(\mathbf{A V} \mathbf{x})^{H}(\mathbf{A V} \mathbf{x})\right\} & =N_{t} N_{r} \sigma_{a}^{2}+\|\mathbf{M}\|_{F}^{2} \\
\mathbf{E}\left\{\mathbf{n}^{H} \mathbf{n}\right\} & =N_{r} \sigma_{n}^{2}
\end{aligned}
$$

and also, that if we consider the original channel matrix,

$$
\mathbf{E}\left\{(\mathbf{A V} \mathbf{x})^{H}(\mathbf{A V} \mathbf{x})\right\}=\sum_{k=1}^{N_{t}} \sigma_{k}^{2}
$$

we can express the signal-to-noise ratio in the following way

$$
S N R=\frac{N_{t} \sigma_{a}^{2}+\frac{\|\mathbf{M}\|_{F}^{2}}{N_{r}}}{\sigma_{n}^{2}}=\frac{\sum_{k=1}^{N_{t}} \sigma_{k}^{2}}{N_{r} \sigma_{n}^{2}}
$$

Defining $\rho_{i}=\frac{\sigma_{i}^{2}}{\sum_{k=1}^{N} \sigma_{k}^{2}}$,

$$
\widehat{S N R_{i}}=\frac{\sigma_{i}}{2 N_{t}\left(1-\alpha^{s}\right) \sigma_{a}^{2}+\sigma_{n}^{2}} \geq \frac{N_{r} \rho_{i}}{2\left(1-\alpha^{s}\right)+\frac{1}{S N R}}
$$

If we assume that the matrix is full rank and we are using all the eigenmodes, then

$$
S N R=\frac{N_{t} E_{s}}{N_{0}}
$$

In the case of 8-PSK and 4 transmit antennas $S N R=$ $12 \frac{E_{b}}{N_{0}}$ so, finally,

$$
\widehat{S N R_{i}} \geq \frac{N_{r} \rho_{i}}{2\left(1-\alpha^{s}\right)+\frac{1}{12\left(E_{b} / N_{0}\right)}}
$$

In Figure 4, the probability of error for $\rho_{i}=\frac{1}{20}$ and $N_{r}=$ 20 , with $\alpha^{s}=0.9,0.95,0.97,0.98,0.99,1$ as a function of $E_{b} / N_{o}(d B)$ is shown.

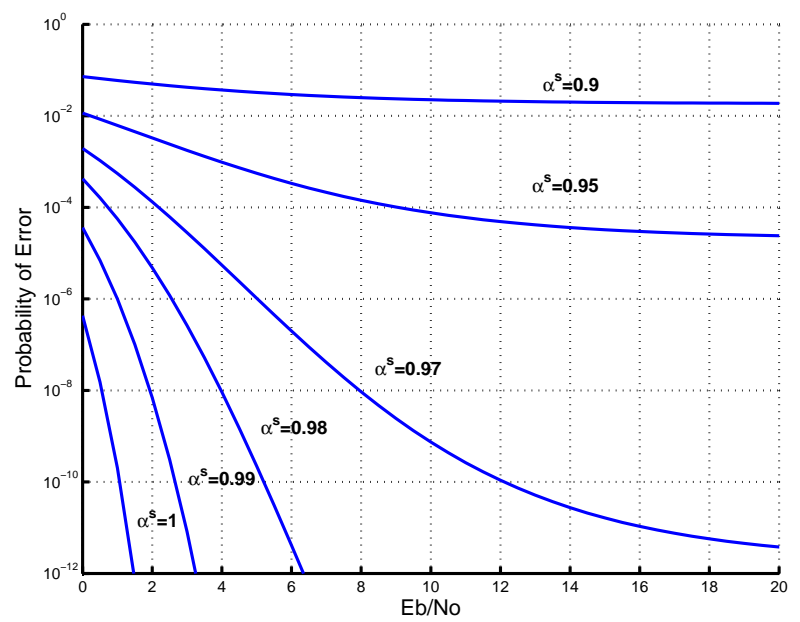

Fig. 4

\section{CONCLUSION}

A study of the impact of channel estimation errors on the performance of SVD-based systems is performed. Bounds relating the Fröbenius norm of the difference between the channel matrix considered and the actual channel matrix with the active eigenmodes' singular values are obtained both from a strict and from a probabilistic viewpoint. For a given estimation error model, those bounds can be used to find the maximum number of active eigenmodes or to determine the maximum elapsed time between two consecutive channel estimations to maintain a certain probability of error.

\section{REFERENCES}

[1] I.E. Telatar, "Capacity of multi-antenna gaussian channels", European transactions on telecommunications, vol. 10, pp. 585-595, Nov-Dec 1999.

[2] G.J. Foschini and M.J. Gans, "On Limits of Wireless Communications in a Fading Environment when Using Multiple Antennas", Wireless Personal Communications, vol. 6, pp. 311-335, March 1998.

[3] Gene H. Golub and Charles F. Van Loan, Matrix Computations, The Johns Hopkins University Press, 1996.

[4] Daniel B. Kilfoyle, James C. Preisig and Arthur B. Baggeroer, "Spatial Modulation Experiments in the Underwater Acoustic Channel". 